\title{
LINEAR/NONLINEAR ADAPTIVE POLYPHASE SUBBAND DECOMPOSITION STRUCTURES FOR IMAGE COMPRESSION
}

\author{
Ömer N. Gerek, A. Enis Çetin \\ Bilkent University, \\ Dept. of Electrical Engineering, \\ Bilkent, Ankara TR-06533, Turkey \\ E-mail: gerek@ee.bilkent.edu.tr
}

\begin{abstract}
Subband decomposition techniques have been extensively used for data coding and analysis. In most filter banks, the goal is to obtain subsampled signals corresponding to different spectral bands of the original data. However, this approach leads to various artifacts in images containing text, subtitles, or sharp edges. In this paper, adaptive filter banks with perfect reconstruction property are presented for such images. The filters of the decomposition structure vary according to the nature of the signal. This leads to higher compression ratios for images containing subtitles compared to fixed filter banks. Simulation examples are presented.
\end{abstract}

\section{INTRODUCTION}

Subband decomposition is widely used in signal processing applications including speech, image and video compression. In most practical cases, the goal is to obtain subband signals corresponding to different spectral bands of the original signal. The frequency content of most audio and visual data are suitable for this kind of frequency selective coding. However, this approach leads to ringing artifacts in image and video signals containing text, subtitles or sharp edges. In this paper, we present Perfect Reconstruction (PR) polyphase filter bank structures in which the filters adapt to the changing input conditions. This leads to higher compression results for images containing sharp edges, text, and subtitles.

The ringing artifacts on the boundaries of subtitles, texts and sharp edges can be removed by using adaptive filters. In this paper, we introduce polyphase filter bank structures with PR property which allow the use of LMS-type linear or nonlinear order statistics based adaptive filters.

This work is supported by NSF Grant No. INT-9406954.
In Section 2, we review the PR polyphase structure concept [1] and present a procedure to make it adaptive. We describe two adaptive polyphase structures one of which contains a fixed anti-aliasing filter for the upper branch and an adaptive prediction filter for the lower branch. In Section 3, multichannel extensions of adaptive filter banks are presented. In Section 4, we present simulation examples and image compression results.

\section{ADAPTIVE PREDICTION FILTERS IN POLYPHASE FORM}

The block diagram of the basic 2-band polyphase subband structure is shown in Fig. 1. In this structure, the

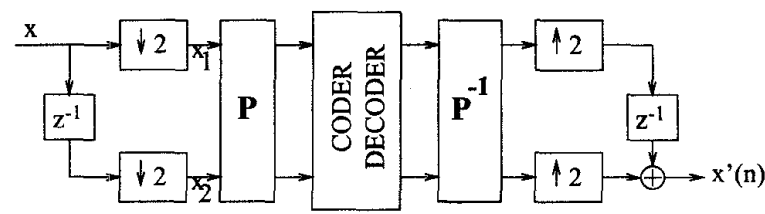

Figure 1: Polyphase decomposition

input polyphase components $x_{1}$ and $x_{2}$ are multiplied by a $2 \times 2$ matrix, $P_{1}$. For perfect reconstruction, the only constraint on this matrix is invertability. One can try to optimize the $\mathbf{P}$ matrix according to the application as long as $\mathbf{P}^{-1}$ exists.

\subsection{The Basic Adaptive Filter Bank Structure}

In this subsection, we describe a specific structure for the $P$ matrix which allows the use of both linear and non-linear adaptive filters while preserving the PR property.

Consider the polyphase filter bank structure shown in Fig 2. This structure has a simple transform matrix: 


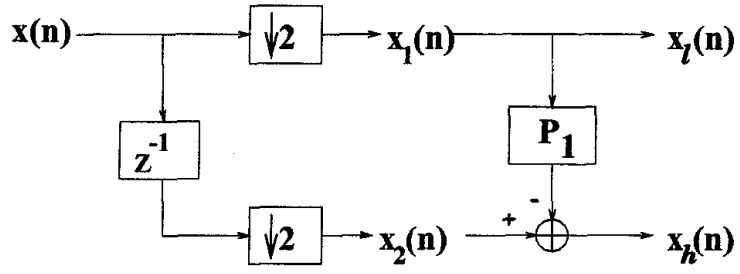

Figure 2: Simple structure analysis stage

$$
\mathbf{P}=\left[\begin{array}{cc}
1 & -P_{1}(.) \\
0 & 1
\end{array}\right]
$$

where the filter $P_{1}$ can be both linear and non-linear. It was shown in [2],[3] that, in coding applications, the Order Statistics (OS) filters and especially the median filter performs better than the linear FIR filters for the images containing sharp variations like text [3].

The inverse matrix is given as:

$$
\mathbf{P}^{-1}=\left[\begin{array}{cc}
1 & P_{1}(.) \\
0 & 1
\end{array}\right]
$$

There is no restriction on the filter $P_{1}$ for perfect reconstruction [2]. This can be proved by observing that the $\mathbf{P}$ matrix is positive-definite regardless of the choice of $P_{1}$ [2],[3]. The resulting synthesis structure is shown in Fig 3.

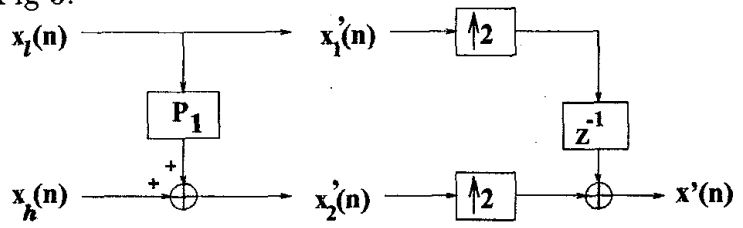

Figure 3: Simple structure synthesis stage

In coding applications, the goal is to remove the correlated portion of the original signal as much as possible. In this way, unnecessary information is eliminated. In this structure, the subsignal $x_{l}$ is simply the downsampled version of the original signal, $x$. Since there is no control over the subsignal $x_{l}(n)$, a good way of obtaining the subsignal, $x_{h}(n)$, is to predict the samples of $x_{2}(n)$ from $x_{1}(n)$ or from $x_{l}(n)$. An adaptive predictor is ideal for image and video signals as they are unstationary in nature. This reasoning leads to the polyphase structure shown in Fig. 4 in which the prediction filter adapts itself to minimize $x_{h}(n)$. This is especially useful when there are sharp transition regions in an image such as subtitles, text and graphics.

In Figure $4, x_{h}(n)$ is obtained by predicting $x_{2}(n)$ from $x_{1}(n)$ as follows:

$$
\hat{x}_{2}(n)=\sum_{k=-N}^{N} a_{k} x_{1}(n-k)=\sum_{k=-N}^{M} a_{k} x(2 n-2 k)
$$

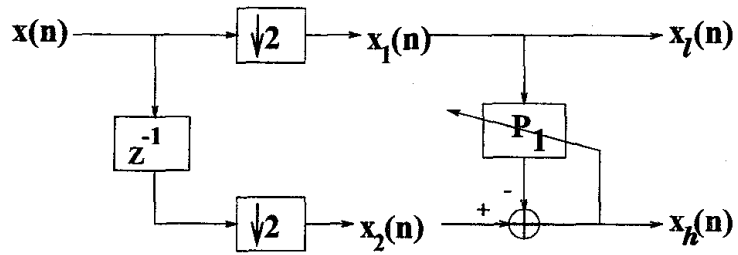

Figure 4: Adaptive structure analysis stage

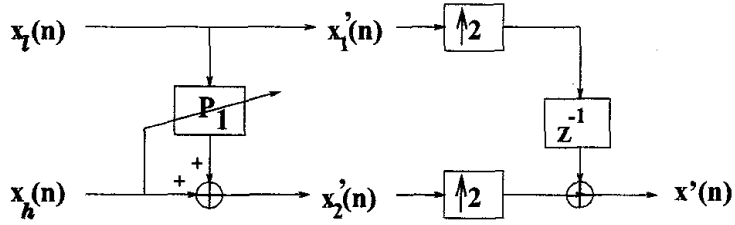

Figure 5: Adaptive structure synthesis stage

where the filter coefficients $a_{k}$ 's are updated using an LMS-type algorithm [4], and the subsignal $x_{h}$ is given by

$$
x_{h}(n)=x_{2}(n)-\hat{x}_{2}(n)
$$

The PR property is preserved in this structure as long as the same adaptation algorithm is used at the encoding and the decoding stage. Since the error signal $x_{h}(n)$ is available both at the encoder and at the decoder, the synthesis stage can adapt the filter $P_{1}$ with the same error signal which provides perfect reconstruction.

The adaptive OS filters can also be used in Figures 4 and 5 . In this case, the input elements are first sorted and the LMS type adaptation is applied to the middle elements of the ordered list.

\subsection{Cascaded Adaptive PR blocks}

The structure described in Subsection 2.1 can be generalized by cascading matrices similar to the matrix in Eq.(1). The analysis and synthesis stages of the cascaded two band decomposition structure can be generated using Equations (1) and (2). The overall cascaded transformation matrix is obtained by multiplying triangular matrices which correspond to basic building blocks as follows:

$\mathbf{P}=\left[\begin{array}{cc}1 & -P_{1}(.) \\ 0 & 1\end{array}\right] \times\left[\begin{array}{cc}1 & 0 \\ G_{1}(.) & 1\end{array}\right] \times\left[\begin{array}{cc}1 & -P_{2}(.) \\ 0 & 1\end{array}\right] \times \cdots$

where the filters $P_{1}, G_{1}, P_{2}, \cdots$ can be linear, nonlinear or adaptive. In this way, the upper and lower branch subsignals can be filtered a number of times. The in- 
verse matrix is given as

$\mathbf{P}^{-\mathbf{1}}=\cdots \times\left[\begin{array}{cc}1 & P_{2}(.) \\ 0 & 1\end{array}\right] \times\left[\begin{array}{cc}1 & 0 \\ -G_{1}(.) & 1\end{array}\right] \times\left[\begin{array}{cc}1 & P_{1}(.) \\ 0 & 1\end{array}\right]$

The synthesis filter bank can be easily reconstructed from $P^{-1}$

\subsection{Adaptive PR Structure with an Anti-Aliasing Filter}

In many applications, multiresolution display of an image is a desirable property. Since $x(n)$ is simply downsampled in the upper branch of Fig 4, the quality of $x_{l}(n)$ is poor due to aliasing.

In order to remove the aliasing, a two stage cascaded $\mathbf{P}$ matrix can be used. The matrix $\mathbf{P}$ should be designed in such a way that the first stage should reduce the aliasing and the second stage should produce a good "high-band" signal.

In a typical QMF structures, the input signal is lowpass filtered before down-sampling to eliminate aliasing. If the low-pass filter is a half-band filter [6], i.e, $H(z)=\frac{1}{2}\left[1+z^{-1} A\left(z^{2}\right)\right]$, then the so called "noble identity" [1] can be used and the filtering operations can be carried out after down-sampling as shown in Fig. 6 .

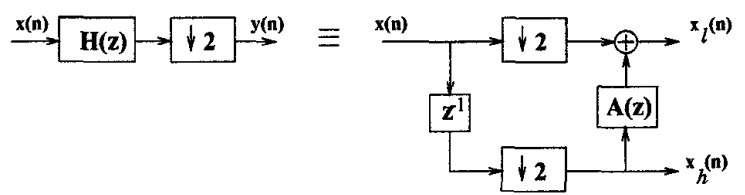

Figure 6: Equivalent structures.

A good choice for the half-band low-pass filter is the Lagrange family [6]. Consider the first two Lagrange filters:

$h_{3}(n)=\cdots 0 \quad 1 / 4 \quad 1 / 2 \quad 1 / 4 \quad 0 \cdots$

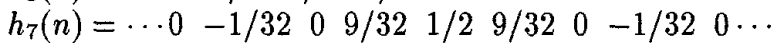
In the first case,

$$
A(z)=\frac{1}{2}+\frac{1}{2} z^{2}
$$

and in the second case,

$$
A(z)=-\frac{1}{16} z^{-2}+\frac{9}{16}+\frac{9}{16} z^{2}-\frac{1}{16} z^{4} .
$$

The second stage of the analysis structure consists of adaptive prediction of subsignal $x_{h}(n)$. In this case, the samples of the low-pass filtered subsignal $x_{l}(n)$ are used to predict $x_{h}(n)$. The overall analysis structure is shown in Fig 7. Perfect reconstruction can be achieved using the synthesis block shown in Fig. 8 .

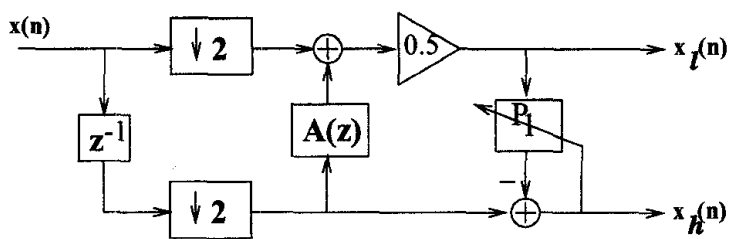

Figure 7: Adaptive Analysis Structure with an AntiAliasing Filter

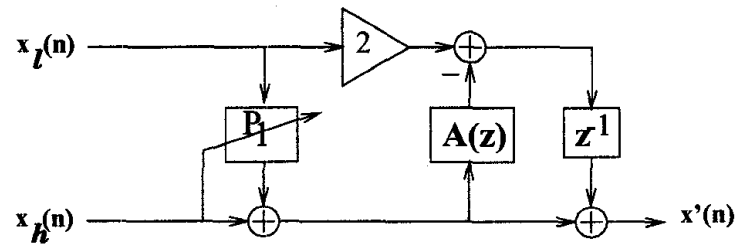

Figure 8: Synthesis stage

Using the PR property of the cascaded structures, the prediction filter $P_{1}$ can be appended to the antialiasing filter and a residual subsignal signal $\left(y_{2}\right)$ can be obtained. The anti-aliased adaptive scheme is illustrated in Fig. 7.

\section{MULTICHANNEL EXTENSION OF THE PR STRUCTURE}

The filter bank structures described in Section 2 can also be extended to handle decompositions to bands other than the powers of two [2]. Consider the multiband decomposition structure shown in Fig 9. In this

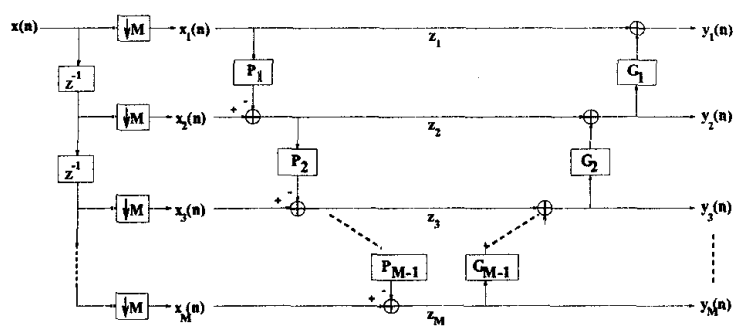

Figure 9: Multi-band analysis structure

figure, an $\mathrm{M}$ band decomposition with two cascaded PR building blocks is illustrated. The PR property of this structure can be proved easily. In the analysis stage,

$$
\begin{aligned}
& z_{1}=x_{1} \\
& z_{i}=x_{i}-P_{i-1}\left(z_{i-1}\right), \quad i=2,3, \ldots, M \\
& y_{i}=z_{i}+G_{i}\left(y_{i+1}\right), \quad i=1,2, \ldots, M-1 \\
& y_{M}=z_{M}
\end{aligned}
$$


and in the synthesis stage, we have

$$
\begin{aligned}
& z_{i}^{\prime}=y_{i}-G_{i}\left(y_{i+1}\right)=z_{i}, \quad i=1, \ldots, M-1 \\
& z_{M}^{\prime}=z_{M} \\
& x_{1}^{\prime}=z_{1} \\
& x_{i}^{\prime}=z_{i}^{\prime}-P_{i-1}\left(z_{i-1}^{\prime}\right)=x_{i}, \quad i=2, \ldots, M
\end{aligned}
$$

The outputs, $x_{i}^{\prime}$, of the synthesis filters are the same as the polyphase components, $x_{i}$, of the analysis filter bank. Notice that there are no restrictions on the filters $P_{i}$ and $G_{i}$ for perfect reconstruction.

\section{SIMULATIONS STUDIES AND CONCLUSIONS}

The proposed structures are used in image compression. The extension to two-dimensions is carried out in a separable manner.

For images that contain text, subtitles or sharp edges, the adaptive filter banks produce better coding results than the conventional Embedded Zerotree Wavelet (EZW) coder [7]. The coding results for the image shown in Fig 10 at $C R=1$ bits/pixel is presented in Table 1. In all the examples, we used the Embedded

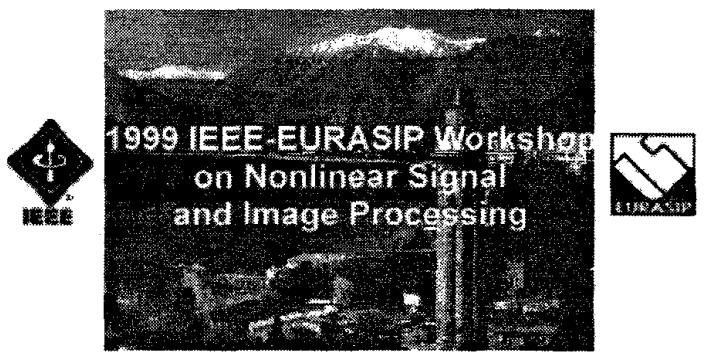

Figure 10: Test image

ZeroTree (EZT) coder to encode the transform coefficients [7]. Due to the characteristics of this coder, the best coding results were obtained by tree-structured two-band decompositions. The first column of the table shows the results without using the anti-aliasing filter stage, and the second column shows the results with the anti-aliasing filter stage. The EZW coder with a fixed filter bank produces a PSNR of $36.10 \mathrm{~dB}$ for this image which is about $1 \mathrm{~dB}$ less than the adaptive filter banks. Furthermore, the adaptive filter banks eliminate the ringing effects which are apparent in the EZW coder as shown in Fig 11. Fig. 11(a) shows the enlarged detail of our encoder output, and Fig. 11(b) shows the EZW output of the same place. Similar coding results are obtained in other images as well.

The $672 \times 560$ "barbara" image was compressed to 1 bits/pixel at a PSNR of $35.80 \mathrm{~dB}$ with the adaptive OS

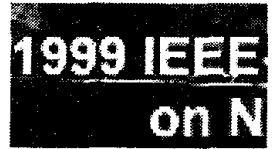

(a)

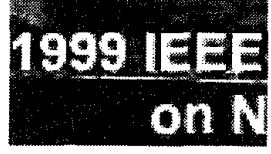

(b)
Figure 11: Details. (a):our method, (b):EZW

\begin{tabular}{|c|c|c|}
\hline$P_{1}$ filter & Plain Downs. & Antialiased Downs. \\
\hline Median & 36.19 & 36.00 \\
\hline Adaptive FIR & 36.80 & 36.76 \\
\hline Adaptive OS & 36.96 & 36.90 \\
\hline
\end{tabular}

Table 1: Experiment results (PSNR) for 5-level decomposition of the test image at $1 \mathrm{bpp}$.

type prediction filter. This PSNR is also comparable to the conventional EZW compression scheme which produces $35.90 \mathrm{~dB}$ PSNR.

It should be noted that the adaptive synthesis filters do not diverge due to the quantization noise at high PSNRs.

\section{REFERENCES}

[1] Gilbert Strang, Wavelets and filter banks, Wellesley - Cambridge Press, Wellesley, MA, 1996.

[2] F. J. Hampson and J. C. Pesquet, "A nonlinear subband decomposition with perfect reconstruction," IEEE Int. Conf. on Image Proc. 1996.

[3] O. Egger, W. Li, and M. Kunt, "High Compression Image Coding Using an Adaptive Morphological Subband Decomposition," Proc IEEE, vol. 83, no. 2, pp.272-287, February 1995.

[4] O. Arıkan, A. E. Çetin, Engin Erzin, 'Adaptive Filtering for non-Gaussian stable processes,' $I E E E$ Signal Processing Letters, vol. 1, No. 11, pp. 163165, November 1994.

[5] P. Salembier, "Adaptive rank order based filters," EURASIP Signal Processing, 27(1):1-25, 1992.

[6] S-M. Phoong, C. W. Kim, P.P Vaidyanathan, R. Ansari, "A new class of two channel biorthogonal filter banks and wavelet bases," IEEE Trans. Signal Proc., Vol.43, No.3, pp. 649-665, March 1995.

[7] J. M. Shapiro, "Embedded Image Coding Using Zerotrees of Wavelet Coefficients," IEEE Trans. Signal Proc., vol. 41, no. 12, pp. 3445 - 3462, Dec. 1993. 